\title{
Protective effect of anisodamine on bleomycin-induced acute lung injury in immature rats via modulating oxidative stress, inflammation, and cell apoptosis by inhibiting the JAK2/STAT3 pathway
}

\author{
Xiaoqi Zhao ${ }^{1}$, Bin Zhao $^{2}$, Yinghao Zhao ${ }^{3}$, Yunfeng Zhang ${ }^{1}$, Min Qian ${ }^{1}$ \\ ${ }^{1}$ Department of Neonatology, the Second Hospital of Jilin University, Changchun, China; ${ }^{2}$ Department of Neurosurgery, the Second Hospital of \\ Jilin University, Changchun, China; ${ }^{3}$ Department of Thoracic Surgery, the Second Hospital of Jilin University, Changchun, China \\ Contributions: (I) Conception and design: M Qian, X Zhao; (II) Administrative support: B Zhao, Y Zhao; (III) Provision of study materials or patients: \\ M Qian, Y Zhang; (IV) Collection and assembly of data: X Zhao; (V) Data analysis and interpretation: X Zhao, B Zhao; (VI) Manuscript writing: All \\ authors; (VII) Final approval of manuscript: All authors. \\ Correspondence to: Min Qian. Department of Neonatology, the Second Hospital of Jilin University, 218 Ziqiang Street, Changchun 130041 , China. \\ Email: qianminjilin@tom.com.
}

Background: Pediatric acute lung injury (ALI) is one of the most common causes of infant mortality. Although lung-protective strategies have developed in recent years, no ALI treatment is currently available. Anisodamine (Ani) is a common drug used to treat gastrointestinal smooth muscle spasm. The protective effects of Ani against acute kidney injury and myocardial injury have been reported. However, the efficacy of Ani on bleomycin (BLM)-induced ALI has not been examined previously. In the present study, we aimed to examine the effects of Ani on bleomycin (BLM)-induced ALI on immature rats.

Methods: The ALI rat model was established by intratracheally administration of BLM. Ani treatment was performed by an intravenous injection at different concentrations. The lung function of each rat was measured, and then lung tissue structures, apoptosis, and collagen deposition were observed by hematoxylineosin staining, terminal deoxynucleotidyl transferase-mediated digoxigenin-dUTP nick-end labeling assay, and Masson's staining, respectively. Enzyme-linked immunosorbent assay was used to detect the levels of inflammatory cytokines. The expression of apoptosis-related proteins and fibrosis-related markers was determined by reverse transcription-polymerase chain reaction and/or Western blot analysis. Finally, the expression levels of Janus tyrosine kinase 2 (JAK2) and signal transducer and activator of transcription 3 (STAT3) were determined.

Results: Our findings indicated that lung function was remarkably decreased in BLM-induced rats, which could be reversed by Ani. Ani treatment increased the levels of antioxidant enzymes, decreased the apoptotic rate and apoptosis-related proteins, and downregulated the expression of fibrosis-related markers. Additionally, Ani treatment also attenuated inflammatory response and suppressed the activation of the JAK2/STAT3 pathway.

Conclusions: Our results demonstrated that Ani had potent activity against BLM-induced ALI in immature rats through inhibiting the JAK2/STAT3 signaling pathway. Our findings provide supporting evidence to further investigate the therapeutic effect of Ani against ALI in children.

Keywords: Anisodamine; acute lung injury (ALI); bleomycin; oxidative stress; inflammation

Submitted Mar 17, 2021. Accepted for publication Apr 28, 2021.

doi: $10.21037 / \mathrm{atm}-21-1750$

View this article at: http://dx.doi.org/10.21037/atm-21-1750 


\section{Introduction}

Acute lung injury (ALI) is a complicated disease linked to the inflammation response that can result in pneumonia, pulmonary edema, and respiratory failure (1). Pediatric ALI is one of the most common causes of infant mortality $(2,3)$. Although lung-protective strategies have developed in recent years, no ALI treatment is currently available. Therefore, finding an effective treatment method to protect and treat pediatric ALI is necessary (4). Bleomycin (BLM), a chemotherapeutic drug for human malignant tumors, is often used to induce ALI in animals $(5,6)$. Therefore, in the present study, BLM was used to establish rat models of ALI.

Previous studies have discovered that the key pathological features of ALI are uncontrolled inflammation and oxidative stress $(7,8)$. Inflammatory responses could result in the significant release of inflammatory mediators, followed by extensive neutrophil infiltration, playing an important role in the pathogenesis of ALI (9). Oxidative stress has been thought to contribute to ALI development (10). In ALI models, superoxide dismutase (SOD) and glutathione (GSH) levels decrease, but lipid peroxidation products increase $(11,12)$; therefore, excessive reactive oxygen species (ROS) will accumulate. Excessive ROS could produce signaling responses, disrupt cellular processes, and exacerbate the degree of tissue injury (13).

The Janus tyrosine kinase 2 (JAK2)/signal transducer and activator of transcription 3 (STAT3) signaling pathway is an important pathway activated by oxidative stress and inflammation. It participates in many physiological processes, including stem cell homeostasis, cell cycle progression, and apoptosis (14). The activation of the JAK2/STAT3 pathway has been observed in ALI $(9,15)$. Many studies have reported that the suppression of the JAK2/STAT3 pathway could ameliorate ALI in rats $(14,16)$. Therefore, the JAK2/STAT3 pathway may function as a potential target in the early intervention of ALI.

Anisodamine (Ani), an extract from the Chinese herb Anisodus tanguticus, is a common drug used to treat gastrointestinal smooth muscle spasm. The protective effects of Ani against acute kidney injury, myocardial injury, and myocardial ischemia/reperfusion injury have been previously reported (17-19). In a randomized controlled trial, patients with traumatic ALI were treated with a high dose of Ani (20). Improved respiratory function of these patients was observed after Ani treatment (20). In You et al.'s study, a model of lipopolysaccharide (LPS)-induced ALI in rats was performed, and Ani effectively alleviated the degree of lung injury induced by LPS (21). However, the efficacy of Ani on BLM-induced ALI has not been examined in previous studies.

In the present study, we used immature rats to establish BLM-induced ALI models, and then investigated the effect of Ani on these models. The potential mechanisms of Ani on ALI were also detected. Our findings provided supporting evidence to further investigate the therapeutic effect of Ani against ALI in children. We present the following article in accordance with the ARRIVE reporting checklist (available at http:// dx.doi.org/10.21037/atm-21-1750).

\section{Methods}

\section{Animals}

Experiments were performed under a project license (No.: SYXK-2018-0015) granted by Institutional Animal Care and Use Committee of Jilin University, in compliance with the Guide for the Care and Use of Laboratory Animals (NIH Publication 86-23, National Academic Press, Washington, DC, USA, 1996). Immature (16-21 days old) Sprague Dawley rats were provided by the Animal Center of Jilin University (Jilin, China). All animals were kept pathogenfree with water and food provided ad libitum, and were raised under conditions at $20-26{ }^{\circ} \mathrm{C}, 40-70 \%$ relative humidity, 15 times/h ventilation and a 12-h light/12-h dark cycle. Ani was purchased from Hangzhou Minsheng Pharmaceutical Group (Hangzhou, China), and BLM was purchased from Dalian Meilun Biotech (Dalian, China).

The present study included two animal studies. The first determined the role of different concentrations of Ani on ALI. Using the random number table, the rats were randomly and equally assigned to the following five groups ( $n=15$ each group): the control group and four BLMinduced groups. At day 0, rats in the BLM-induced groups received the treatment of intratracheal instillation of BLM, as described previously (a single dose of $2.5 \mathrm{mg} / \mathrm{kg}$ in $0.4 \mathrm{~mL}$ saline) (22). The rats in the control group received the treatment of $0.4 \mathrm{~mL}$ saline instead of BLM. Rats in the four BLM-induced groups were then treated by intraperitoneal injection of various concentrations of Ani $(0,5,10$, and $20 \mathrm{mg} / \mathrm{kg}$ ) once a day for 7 consecutive days (days 1-7).

AG490, a JAK2 inhibitor, was used in the other animal study; $3.5 \%$ dimethyl sulfoxide (DMSO) was used to dissolve AG490 (Sigma, St Louis, MO, USA). To eliminate the influence of DMSO on the JAK2/STAT3 pathway, 
20 rats were randomly divided into the following four groups ( $\mathrm{n}=5$ each group): the control group, BLM group, BLM+DMSO group, and BLM + DMSO + AG490 group. AG490 was administered intraperitoneally at a single dose of $20 \mathrm{mg} / \mathrm{kg}$ (in $0.4 \mathrm{~mL}$ of DMSO) $30 \mathrm{~min}$ prior to BLM administration (day 0 ). Then, 40 rats were randomly divided into the control group, BLM group, BLM + AG490 group, and BLM + AG490 + Ani group, with 10 rats in each group. The administration of AG490 and Ani were performed as previously mentioned. Rats in each group were sacrificed by cervical dislocation on day 8 after lung function test, and the lung tissues and blood samples were collected for further analysis.

\section{Lung function test}

After the final dose of Ani, rats in each group ( $n=6)$ were anesthetized. A polyethylene cannula was then inserted. Rats were attached to a ventilator (Servo-I; MAQUET, Solna, Sweden) after pancuronium bromide $(2 \mathrm{mg} / \mathrm{kg}$; Sigma, USA) administration. Lung function was analyzed by a computer-controlled piston ventilator (flexiVent system; SCIREQ, Montreal, QC, Canada). Rats were mechanically ventilated with a tidal volume of $7 \mathrm{~mL} / \mathrm{kg}$. Airway resistance and elastance values were measured automatically.

\section{Measurement of oxidative stress markers}

To obtain supernatants, the lung tissues were centrifuged at $10,000 \times \mathrm{g}$ for $15 \mathrm{~min}$ at $4{ }^{\circ} \mathrm{C}$. Levels of oxidative stress markers, such as lactate dehydrogenase (LDH), SOD, and GSH, in supernatants were detected by commercial kits (Nanjing Jiancheng, Nanjing, China), following the manufacturer's instructions.

\section{Histological analyses, terminal deoxynucleotidyl transferase-mediated digoxigenin-dUTP nick-end labeling (TUNEL) assay, and Masson's staining}

The upper right lobe of the lungs in each group was isolated, washed, and fixed with $4 \%(\mathrm{v} / \mathrm{v})$ paraformaldehyde. The tissues were then embedded in paraffin, sectioned at 5 - $\mu \mathrm{m}$ thickness, and stained with hematoxylin-eosin (HE) solution.

Cell apoptosis was determined by TUNEL staining using TUNEL kits (Beyotime, Shanghai, China). Cell apoptosis was determined by ImageJ software (Bethesda, MD, USA).
Masson's kits (Fuzhou Maixin Biotechnology, Fuzhou, China) were used to assess alveolitis and fibrosis of lung tissues, according to the manufacturer's protocol. The quantitative histological analysis of fibrotic changes was evaluated by the Ashcroft scale (23).

\section{Bronchoalveolar lavage fluid (BALF) and blood analysis}

Blood samples were centrifuged $(1,800 \times \mathrm{g})$ for $10 \mathrm{~min}$ at $4{ }^{\circ} \mathrm{C}$ to separate serum. BALF was obtained by intratracheal instillation of $0.9 \%$ saline solution. The BALF supernatant was then centrifuged at $800 \times \mathrm{g}$ for $10 \mathrm{~min}$ at $4{ }^{\circ} \mathrm{C}$ to obtain the cell pellet. The total leukocytes in BALF was counted using a Neubauer chamber hemocytometer (Beckman Coulter, Brea, CA, USA) after the cell pellet was resuspended in $500 \mu \mathrm{L}$ normal saline.

Interleukin (IL)-1 $\beta$, IL-4, IL-6, IL-10 and tumor necrosis factor- $\alpha(\mathrm{TNF}-\alpha)$ levels in serum and BALF were measured by enzyme-linked immunosorbent assay using commercial kits (Beyotime, China).

\section{Reverse transcription-polymerase chain reaction}

Total RNA was extracted with TRIzol reagent (Omega BioTek, Norcross, GA, USA), according to the manufacturer's instructions. cDNA was synthesized using a cDNA synthesis kit (TaKaRa Bio, Dalian, China). Primer sequences (Qinke, Beijing, China) are shown in Table 1. Relative expression gene levels were measured by a fluorescence quantitative PCR instrument (Bio-Rad, Hercules, CA, USA) and analyzed using the $2^{-\Delta \Delta C t}$ method.

\section{Western blot}

Radioimmunoprecipitation assay lysis buffer was used to extract total protein from lung tissues. The extracted proteins were then transferred to polyvinylidene difluoride membranes after separation by sodium dodecyl sulfatepolyacrylamide gel electrophoresis gel. After blocking for $1 \mathrm{~h}$, blots were incubated with primary antibodies overnight at $4{ }^{\circ} \mathrm{C}$. Specific primary antibodies against fibronectin [1:2,000; Cell Signaling Technology (CST), Danvers, MA, USA], $\alpha$-smooth muscle actin ( $\alpha$-SMA; 1:1,000; CST), caspase-3 (1:1,000; CST), B-cell lymphoma 2 (Bcl-2; 1:1,000; CST), Bcl2-associated X protein (Bax; 1:1,000; CST), transforming growth factor $\beta$ (TGF- $\beta ; 1: 11,000$; CST), JAK2 (1:1,000; CST), phosphor (p)-JAK2 (1:1,000; CST), STAT3 (1:1,000; CST), and p-STAT3 $(1: 1,000$; 
Table 1 Primer sequences used for the reverse transcription quantitative-polymerase chain reaction

\begin{tabular}{ll}
\hline Gene & Primer sequence (5'-3') \\
\hline Ki67 & F: CGGCGAGCCTCAAGAGATA \\
R: CGTGCTGTTCTACATGCCC & F: CCTTGCTGGGCAACCTGGA \\
& R: GCCTTGCACAACCCTCCCTT \\
TGF- $\beta$ & F: TACTGCTTCAGCTCCACAGAGA \\
& R: CAGACAGAAGTTGGCATGGTAG \\
Fibronectin & F: TGACAACTGCCGTAGACCTGG \\
& R: TACTGGTTGTAGGTGTGGCCG \\
$\alpha$-SMA & F: GACCGAATGCAGAAGGAGAT \\
$\beta$-actin & R: CCACCGATCCAGACAGAGTA \\
& F: TCACCCACACTGTGCCCATCTATGA \\
& R: ATCGGAACCGCTCATTGCCGATAG \\
\hline
\end{tabular}

TGF- $\beta$, transforming growth factor $\beta ; \alpha$-SMA, $\alpha$-smooth muscle actin.

CST) were incubated. After that, membranes were incubated with secondary antibodies (1:2,000; Wuhan Boster Biological Technology, Wuhan, China). $\beta$-actin was used as the internal control.

\section{Statistical analysis}

Data were analyzed by SPSS version 25.0 (IBM, Armonk, NY, USA). Quantitative data were expressed as mean \pm standard deviation and analyzed by one-way analysis of variance. $\mathrm{P}<0.05$ were considered statistically significant.

\section{Results}

\section{Effect of Ani on lung function in BLM-induced ALI rats}

Compared with the control, rats in the BLM group had higher airway resistance and elastance $(\mathrm{P}<0.01)$, indicating that BLM could inhibit lung function. However, Ani treatment reversed the inhibitory effect of BLM on lung function in a dose-dependent manner. Rats in the BLM + Ani $10 \mathrm{mg} / \mathrm{kg}$ group and BLM + Ani $20 \mathrm{mg} / \mathrm{kg}$ group had much lower resistance and elastance values than those in the BLM group $(\mathrm{P}<0.01)$ (Figure 1A,B).
Effect of Ani on oxidative stress in BLM-induced ALI rats

To evaluate the protective effect of Ani on oxidative stress, antioxidant enzyme activity was detected. Compared with the control, GSH and SOD levels decreased in the BLM group. However, this effect was reversed by treatment with Ani. The BLM + Ani $10 \mathrm{mg} / \mathrm{kg}$ group and BLM + Ani $20 \mathrm{mg} / \mathrm{kg}$ group had much higher GSH and SOD levels than the BLM group, respectively $(\mathrm{P}<0.05)$ (Figure $1 C, D)$. Furthermore, LDH was significantly higher in the BLM group than in the control group $(\mathrm{P}<0.05)$. Ani treatment could clearly prevent increase in LDH content (Figure 1E).

\section{Effect of Ani on cell proliferation and cell apoptosis}

HE staining showed that rats in the BLM-treated group exerted obvious features of ALI, with pathological changes including inflammatory cell infiltration, alveolar septum thickening, and perivascular space edema. However, these pathological changes induced by BLM were reversed by Ani treatment (Figure 2A).

Apoptosis of alveolar epithelial cells is a feature of ALI. As expected, a high apoptotic rate was observed in the BLM group. Ani treatment significantly attenuated BLMinduced cell apoptosis. The apoptotic rate in the BLM + Ani $10 \mathrm{mg} / \mathrm{kg}$ group or BLM + Ani $20 \mathrm{mg} / \mathrm{kg}$ group was much lower than that in the BLM group $(\mathrm{P}<0.05)$ (Figure 2B). Ki-67 and survivin are markers of cell proliferation. Compared with the control, the mRNA levels of $\mathrm{Ki}-67$ and survivin were much lower in the BLM group $(\mathrm{P}<0.05)$, indicating that BLM could in inhibit cell proliferation. However, Ani treatment could reverse the inhibition effect caused by BLM (Figure 2C). Furthermore, $\mathrm{BLM}$ significantly increased $\mathrm{Bax} / \mathrm{Bcl}-2$ and cleaved caspase-3/caspase-3 ratios (Figure 2D). Ani treatment could prevent the increase of these two ratios (Figure 2D).

\section{Effect of Ani on lung fibrosis in rats with ALI}

As shown in Figure $3 A$, the control group had very few thin filamentous fiber deposition, while the BLM group had large amounts of fiber deposition. In the present study, the Ashcroft score was used to quantify lung fibrosis in each group. Compared with the BLM group, the degrees of fiber deposition in three Ani treatment groups decreased (Figure 3B). Furthermore, compared with the 
A

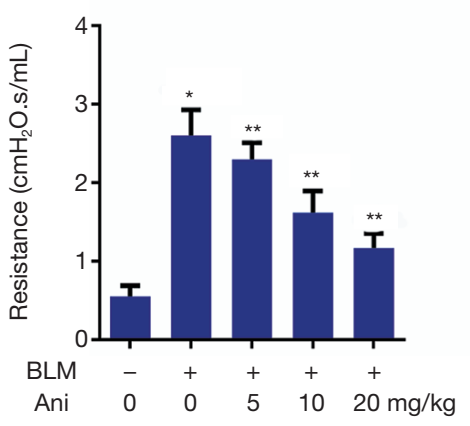

C

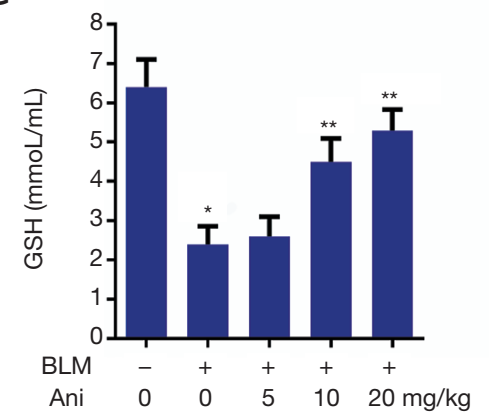

B

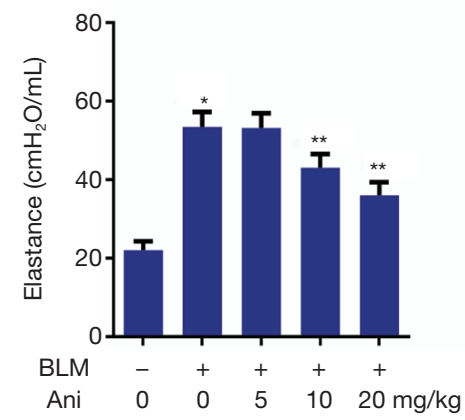

$E$

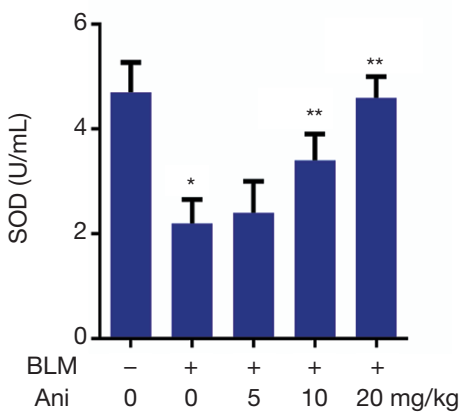

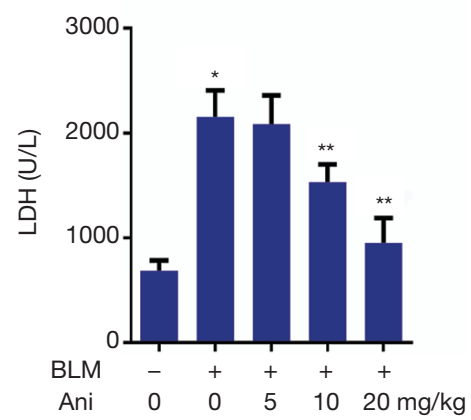

Figure 1 Anisodamine (Ani) increased lung function and reduced oxidative stress induced by bleomycin (BLM). (A) Airway resistance value measured in each group; (B) lung elastance value measured in each group; (C) glutathione (GSH) activity in lung tissue homogenate; (D) superoxide dismutase (SOD) activity in lung tissue homogenate; (E) lactate dehydrogenase (LDH) level in lung tissue homogenate. *, $\mathrm{P}<0.05$ vs. control group; ** $\mathrm{P}<0.05$ vs. BLM group.

control group, the mRNA levels of TGF- $\beta$, fibronectin, and $\alpha$-SMA were markedly increased in the BLM group $(\mathrm{P}<0.05)$. In contrast, Ani treatment could prevent an increase of these markers caused by BLM (Figure 3C). Similarly, the western blot analysis showed that Ani could also diminish the BLM-induced increase in protein levels of TGF- $\beta$, fibronectin, and $\alpha$-SMA in lung tissues (Figure 3D,E,F,G).

\section{Effect of Ani on inflammation in rats with ALI}

BLM treatment significantly increased the levels of IL6 , IL-1 $\beta$, and TNF- $\alpha$ in BALF and serum compared with the control (Figure 4A,B,C). Ani administration was found to decrease these factor levels (Figure $4 A, B, C$ ). In contrast, BLM treatment did not cause significant changes in IL-4 and IL-10 levels in BALF and serum. However, the treatment of Ani at 10 and $20 \mathrm{mg} / \mathrm{kg}$ resulted in significant increases of IL-4 and IL-10 levels in the BLM models (Figure 4D,E). As shown in Figure $4 F$ and $G$, BLM increased the number of total leukocytes and neutrophils in BALF compared with the control group $(\mathrm{P}<0.05)$, indicating that leukocytes and neutrophils accumulated and were activated during the process of BLM-induced ALI. However, Ani treatment abrogated this effect.

\section{Effect of Ani on the FAK2/STAT3 signaling pathway}

JAK2/STAT3 is a well-known pathway activated by oxidative stress and pro-inflammatory factors involved in the development of ALI $(14,16)$. Therefore, we tested whether Ani, which can ameliorate BLM-induced ALI, is associated with the JAK2/STAT3 signaling pathway. The results indicated that BLM treatment significantly induced the increase of p-JAK2 and p-STAT3 levels. In contrast, Ani treatment had an effect against BLM on the expression of p-JAK2 and p-STAT3. Therefore, the p-JAK2/JAK2 and p-STAT3/STAT3 ratios in the BLM group were highest among 5 groups. Compared with the BLM group, decreased values of p-JAK2/JAK2 and p-STAT3/STAT3 ratios were observed in the Ani treatment 
A

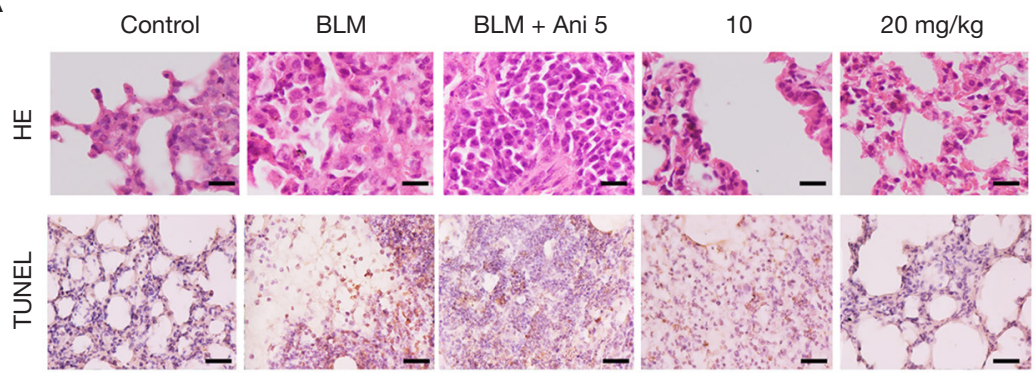

C

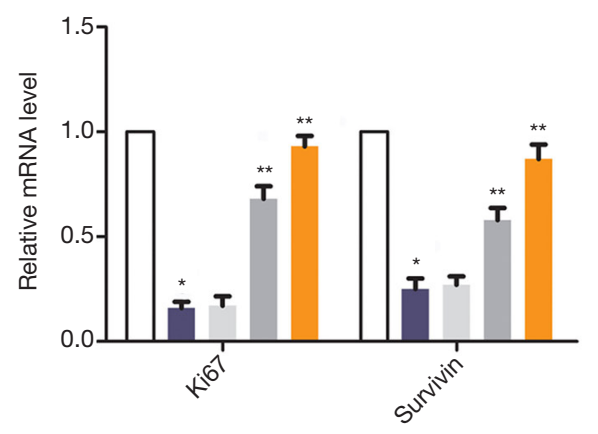

D

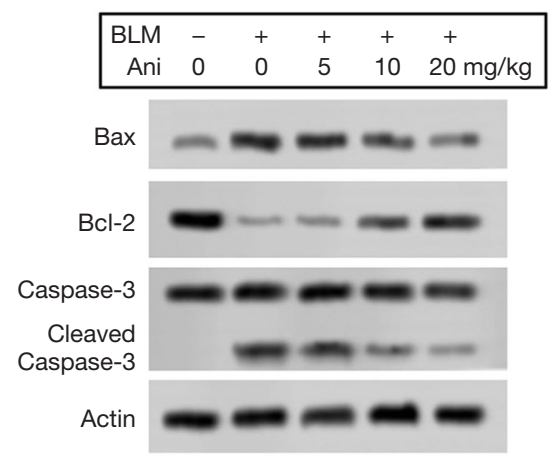

B

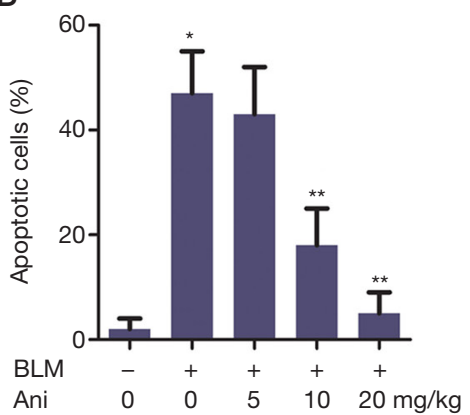

Figure 2 Anisodamine (Ani) inhibited cell proliferation and cell apoptosis in rats with acute lung injury. (A) Pathological changes of lung tissue observed after hematoxylin-eosin (HE) staining (magnification 400x, scale bar $=25 \mu \mathrm{m}$ ) and terminal deoxynucleotidyl transferasemediated digoxigenin-dUTP nick-end labeling (TUNEL) assay (magnification 400×, scale bar $=25 \mu \mathrm{m}$ ); (B) comparison of apoptotic rate in each group; (C) mRNA levels of Ki-67 and survivin in each group measured by reverse transcription-polymerase chain reaction; (D) expression of apoptosis-related proteins in each group measured by western blot. *, $\mathrm{P}<0.05$ vs. control group; **, $\mathrm{P}<0.05$ vs. BLM group; Bax, Bcl2-associated X protein; Bcl-2, B-cell lymphoma 2.

groups (Figure 5A), indicating that Ani could prevent BLMinduced JAK2/STAT3 pathway activation.

\section{Comparison of Ani and AG490 on ALI in a BLM-treated rat model}

AG490 was used to further detect the role of Ani on the JAK2/STAT3 pathway. The rats were treated with the control, BLM, BLM + DMSO, and BLM + DMSO + AG490. The ratios of p-JAK2/JAK2 and p-STAT3/ STAT3 were not significantly different between the BLM and BLM + DMSO groups, indicating that DMSO could not affect the JAK2/STAT3 pathway (Figure $5 B$ ). The rats were then divided into the following four groups: control, BLM, BLM + AG490, and BLM + AG490 + Ani. Compared with the BLM group, the p-JAK2/JAK2 and 
A

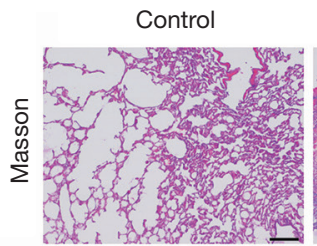

BLM

BLM + Ani 5

10

$20 \mathrm{mg} / \mathrm{kg}$
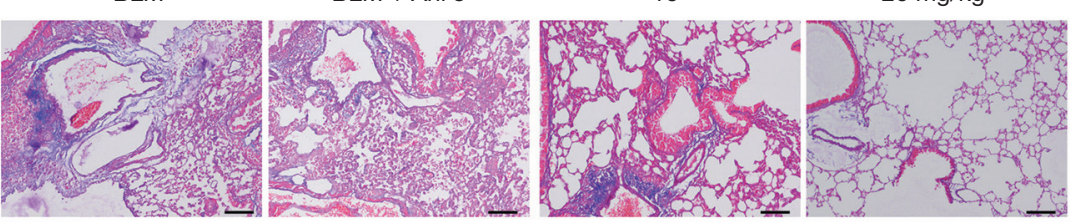

B
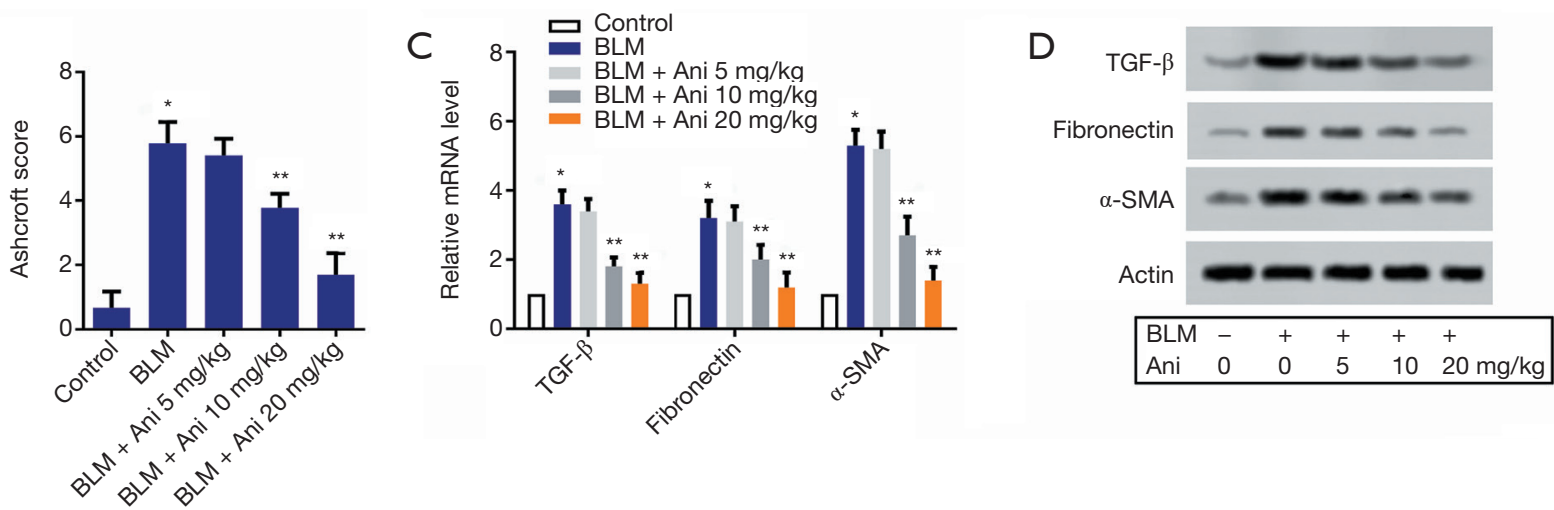

E

F
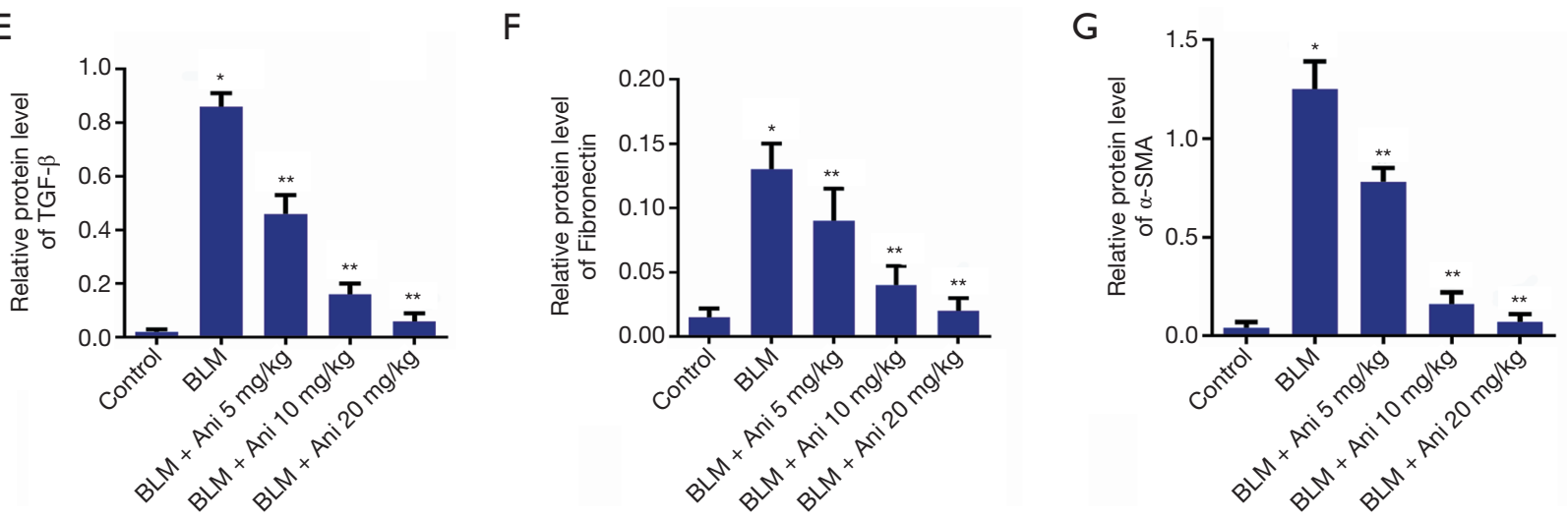

Figure 3 Anisodamine (Ani) inhibited lung tissue fibrosis in rats with acute lung injury. (A) Representative images of Masson staining in each group (magnification 100x); collagen is stained in blue and cells in red (scale bar =100 $\mu \mathrm{m}$ ); (B) quantification of lung fibrosis shown as an Ashcroft score; (C) mRNA levels of transforming growth factor $\beta$ (TGF- $\beta$ ), fibronectin, and $\alpha$-smooth muscle actin ( $\alpha$-SMA) measured by reverse transcription-polymerase chain reaction; (D) representative images of western blot analysis of TGF- $\beta$, fibronectin, and $\alpha$-SMA; (E) protein levels of TGF- $\beta$ measured by western blot; (F) protein levels of fibronectin measured by western blot; (G) protein levels of $\alpha$-SMA measured by western blot. *, $\mathrm{P}<0.05$ vs. control group; **, $\mathrm{P}<0.05$ vs. bleomycin (BLM) group.

p-STAT3/STAT3 ratios in the BLM + AG490 group were much lower $(\mathrm{P}<0.05)$. Furthermore, the $\mathrm{p}-\mathrm{JAK} 2 / \mathrm{JAK} 2$ and p-STAT3/STAT3 ratios in the BLM + AG490 + Ani group were lowest among the 3 BLM-treated groups, with a statistical significance compared with the BLM + AG490 group $(\mathrm{P}<0.05)$ (Figure $5 C)$. These results further indicated that Ani could prevent the activation of the JAK2/STAT3 pathway induced by BLM.

As shown in Figure 5D, the obvious pathological features of ALI were observed in rats in the BLM group.
However, the degree of ALI in rats in the BLM + AG490 group and BLM + AG490 + Ani group were alleviated (Figure $5 D$ ). The rate of apoptotic cells and the expressions of TGF- $\beta$, fibronectin, and $\alpha$-SMA in the BLM + AG490 group were much lower than those in the BLM group $(\mathrm{P}<0.05)$ (Figure 5E,F). Furthermore, the BLM + AG490 group also had a lower LDH level and higher SOD level compared with the BLM group $(\mathrm{P}<0.05)$ (Figure $5 G, H)$. As expected, the serum IL-6 level decreased in the BLM + AG490 group compared with the BLM group $(\mathrm{P}<0.05)$ 

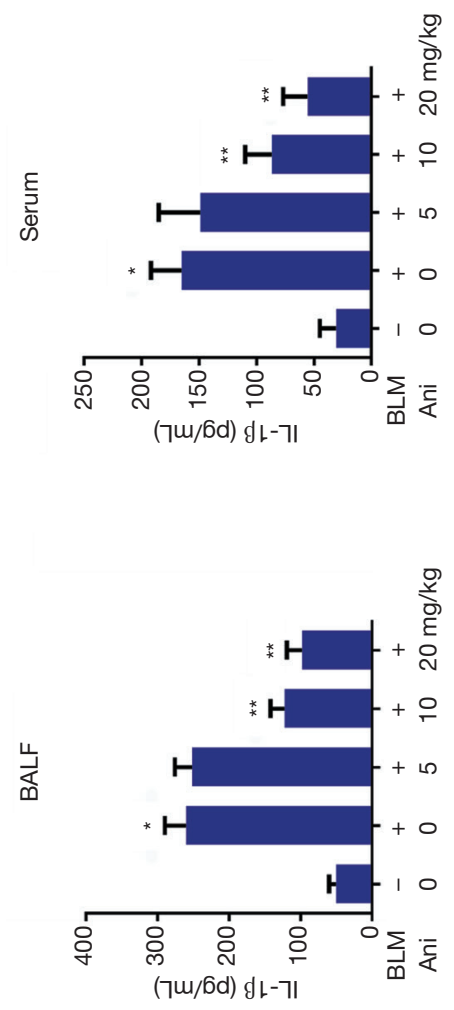

$\infty$
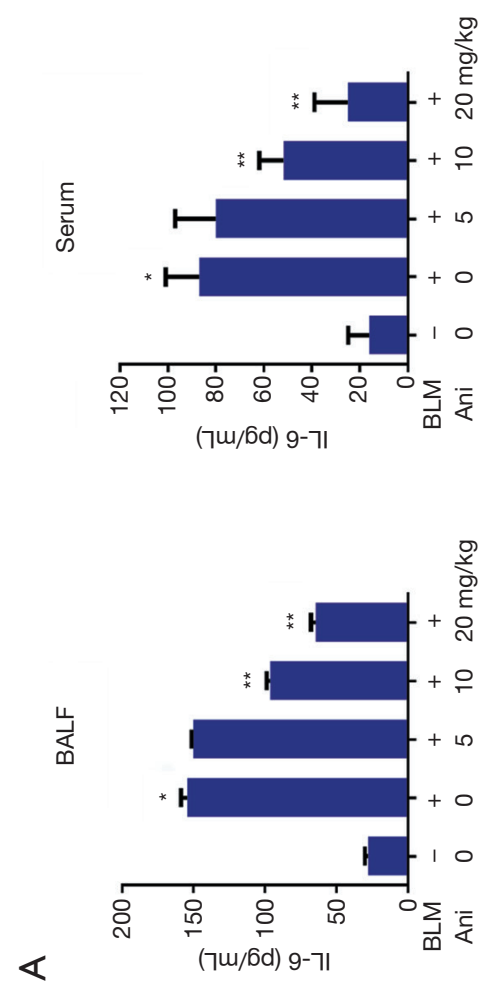
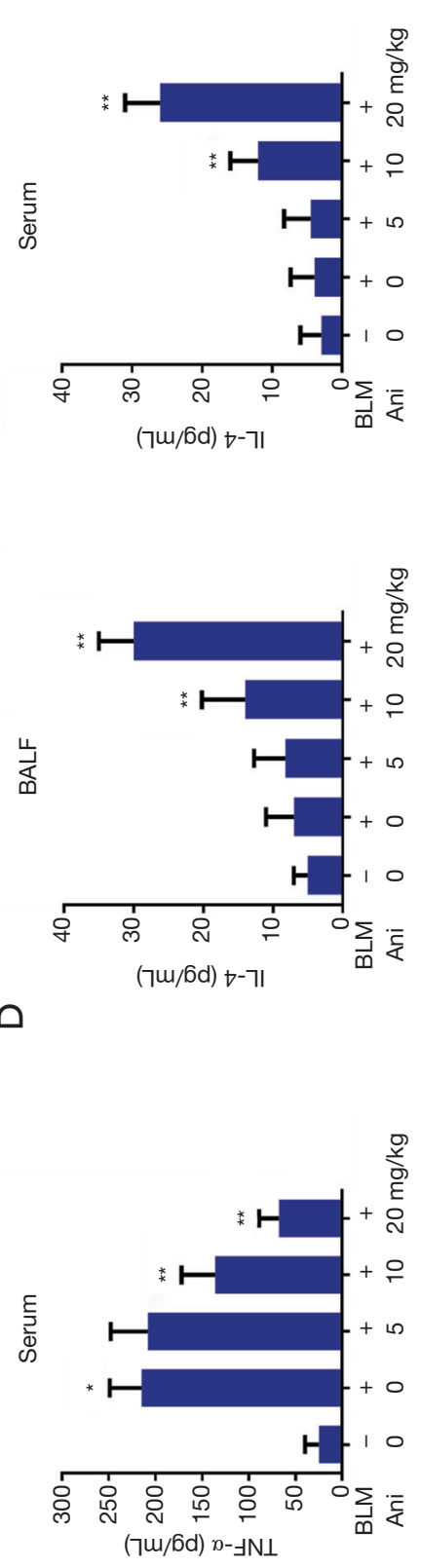

○

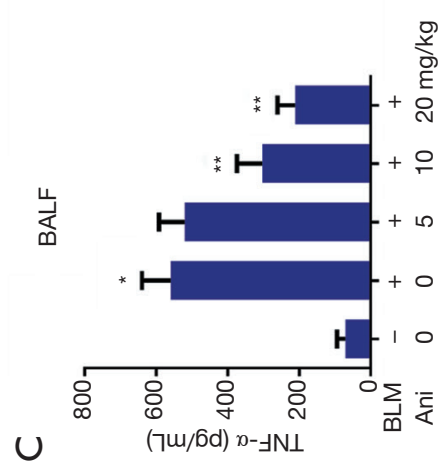

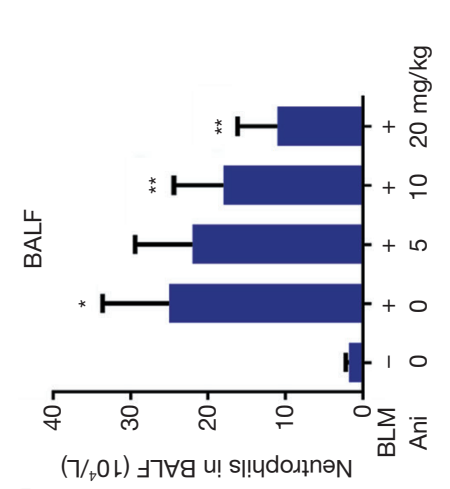

$\circlearrowleft$

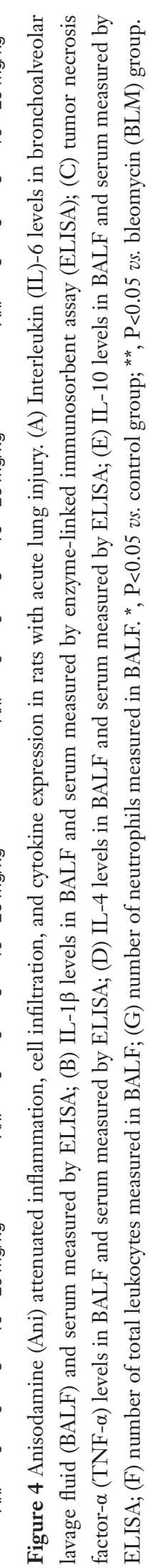




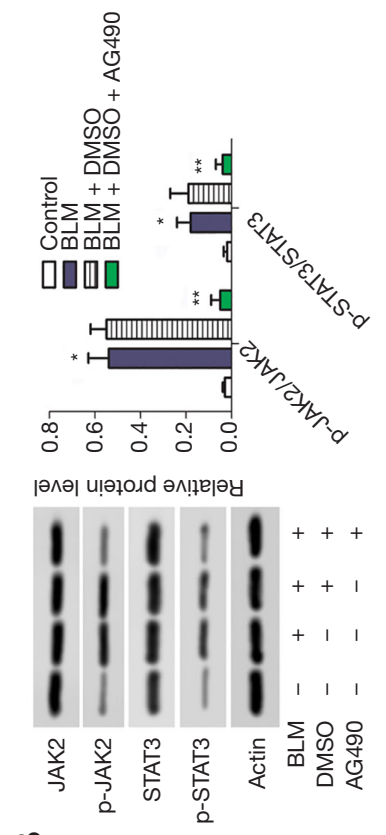

$\infty$

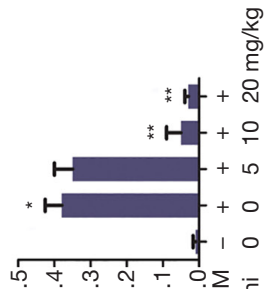

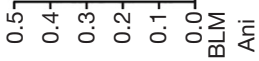

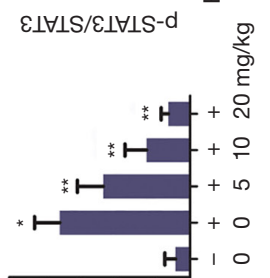

芯 zXy

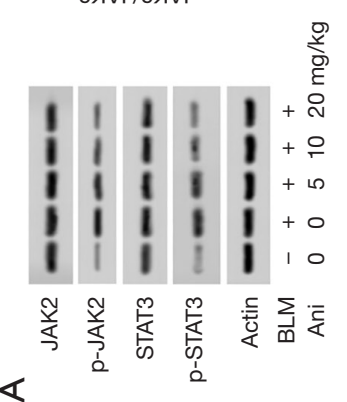

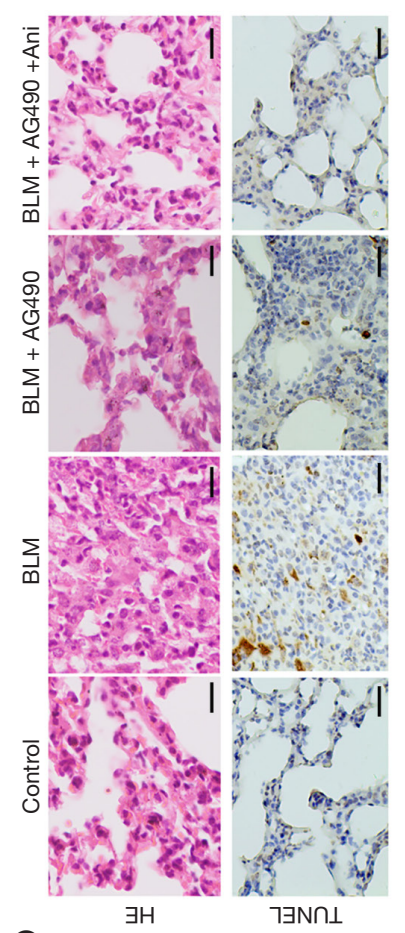

口
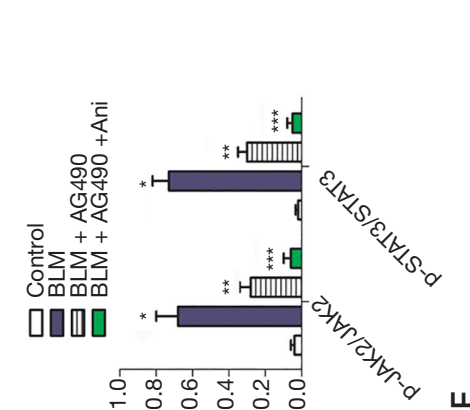

U

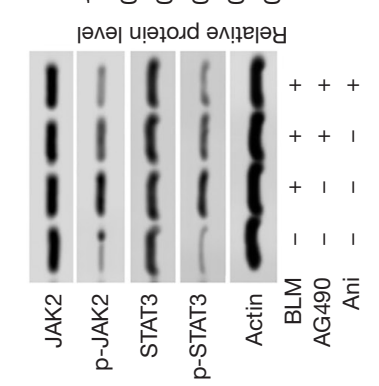

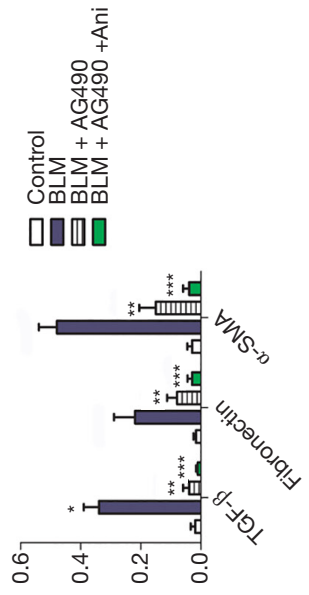

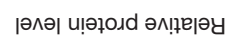
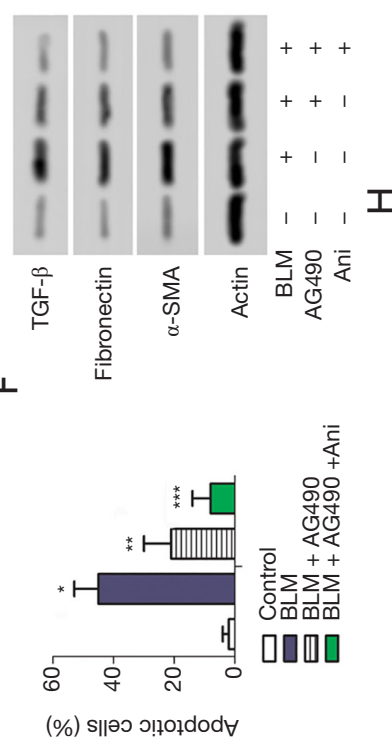

ш

I
\%) s॥əə ग!ฺ०łdod $\forall$

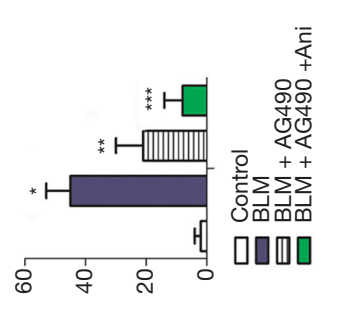

$\cup$

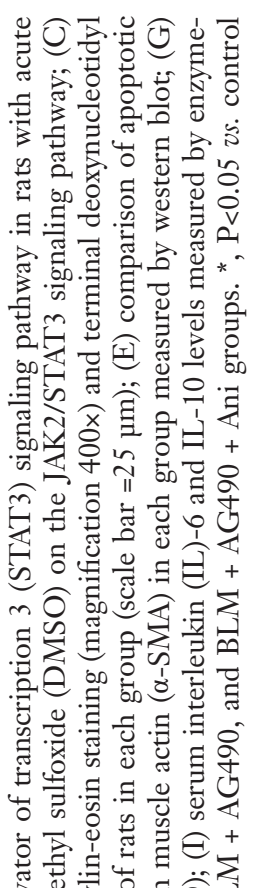

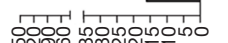

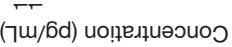

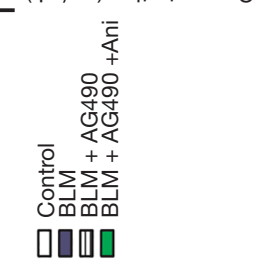

प्याप
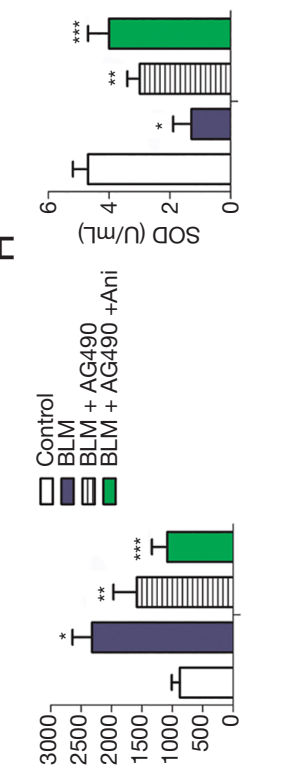

( $7 / \mathrm{\cap}) \mathrm{HO}$
党

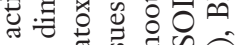

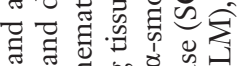

ปัँ

娄语的

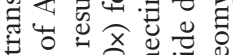

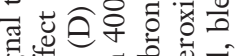

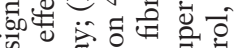

तิ

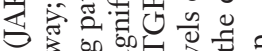

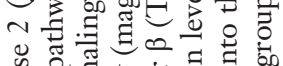

㣰

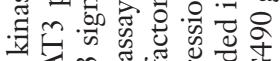

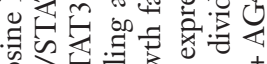

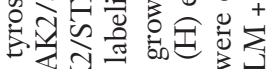

造造

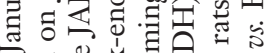

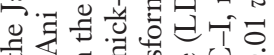

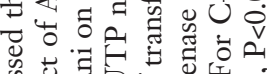

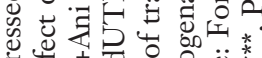

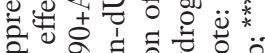

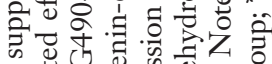

至

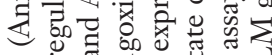

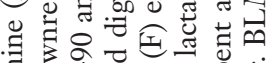

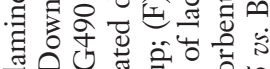

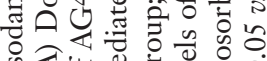

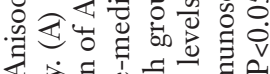

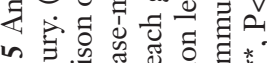

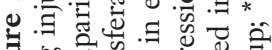

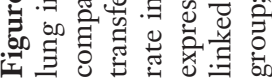


(Figure 5I). The serum IL-10 level had a reverse trend $(\mathrm{P}<0.05)$ (Figure 5I). These data indicated that inhibiting the JAK2/STAT3 pathway could attenuate apoptosis, fibrosis, oxidative stress, and inflammation in BLMinduced ALI. Moreover, this effect was reinforced by Ani treatment (Figure 5E,F,G,H,I), further suggesting that Ani exhibited a protective effect on BLM-induced ALI via inactivating the JAK2/STAT3 pathway.

\section{Discussion}

In the current study, BLM-treated immature rats presented with typical characteristics of ALI, such as decreased lung function, increased cell apoptosis, and increased fiber deposition. However, Ani could reverse the lung damage effect of BLM. Our results showed that Ani could suppress oxidative stress and inflammatory responses, inhibit cell apoptosis and tissue fibrosis, and modulate the JAK2/ STAT3 pathway in BLM-induced ALI. Therefore, our findings indicated that Ani had a protective effect on BLMinduced ALI in immature rats.

In previous studies, Ani was found to have a promising protective effect against various diseases, including myocardial ischemia/reperfusion injury and kidney injury $(18,19)$. In LPS-induced ALI, Ani was found to eliminate injury via reducing IL-17A and IL-17F levels (21). In the present study, the typical pathological changes of BLMinduced ALI in immature rats were observed by HE staining. Ani reversed these pathological changes and even improved lung function of BLM-induced ALI models, indicating that Ani also had a protective effect on BLMinduced ALI.

It has been reported that ALI is a complex syndrome with excessive inflammation and oxidative stress response $(24,25)$. The inflammatory response, which includes the recruitment of inflammatory cells (e.g., neutrophils, lymphocytes, and macrophages) to the lung and the release of chemokines, such as IL- 6 , IL- $1 \beta$, and TNF- $\alpha(26,27)$, is one of the typical features in the early stage of ALI. In the present study, the expression of IL- 6, IL-1 $\beta$, and TNF- $\alpha$ was significantly elevated in BALF and serum in BLM-induced ALI. Ani administration was found to prevent BLM-induced increase of these inflammatory factors. Interestingly, the expression of IL-4 and IL-10, two main anti-inflammatory mediators involved in regulating inflammatory responses (28), were also increased by Ani. The effect of Ani on pro-inflammatory and antiinflammatory chemokines indicated that Ani could inhibit inflammatory responses in BLM-induced ALI, which was consistent with the results obtained from other studies (18). Recruitment of neutrophils into the lung tissues is an important feature of ALI (26). In the present study, our results indicated that Ani significantly prevented neutrophil infiltration into the lung tissues in a BLM-induced ALI rat model. However, the specific mechanisms of this effect should be determined in future studies.

Oxidative stress plays an important role in the development of ALI (29). In previous studies, Ani was found to exhibit antioxidant activity in kidney injury and myocardial dysfunction $(18,19)$. To assess the effect of Ani on oxidative stress in BLM-induced ALI, SOD, GSH, and LDH levels in lung tissues were measured. SOD and GSH are key endogenous antioxidant enzymes, while LDH is regarded as a biomarker of systemic inflammation (30). In the current study, Ani increased SOD and GSH activity and decreased LDH levels in BLM-induced ALI, suggesting that Ani might protect against ALI by enhancing endogenous antioxidant defense capacity and reducing ROS-induced oxidative stress.

Alveolar epithelial cell apoptosis is another feature of ALI (12). In the present study, a high apoptotic rate in BLM-induced ALI was observed. Ani treatment attenuated BLM-induced cell apoptosis significantly. Bcl-2, in contrast of Bax, is an anti-apoptotic protein in the mitochondrial apoptotic pathway (31). Caspase-3 is an important protein participating in the execution phase of cell apoptosis (32). In the present study, Bax and cleaved caspase-3 were downregulated, while Bcl-2 was upregulated after Ani treatment, suggesting that Ani may have a role in inactivating the apoptotic pathway.

Fibrosis is one of the most important characteristics of ALI. A previous study conducted by Luo and Zhou revealed antifibrotic activity of Ani in vivo using liver fibrosis models (33). In the present study, we also found that Ani significantly decreased the degree of fiber deposition in lung tissues in BLM-induced ALI. TGF- $\beta$ exerted its fibrotic activity through the stimulation of fibroblast proliferation and extracellular matrix (ECM) synthesis (including fibronectin) (34), thereby promoting ECM-producing cells to express $\alpha$-SMA. $\alpha$-SMA is the most characteristic marker of pathological fibroblasts (34). Therefore, the expression of TGF- $\beta$, fibronectin, and $\alpha$-SMA increase in the development of organ fibrosis. In the present study, mRNA and protein levels of these fibrosis markers were markedly increased in BLM-induced ALI, as expected. In contrast, Ani treatment prevented the increase of these 
markers caused by BLM, indicating that Ani could inhibit the fibrosis formation pathway.

The JAK2/STAT3 is a pathway activated by oxidative stress and pro-inflammatory factors (35). JAK2 is activated through phosphorylation, subsequently resulting in the phosphorylation and dimerization of STAT3 (36). It has been reported that the JAK2/STAT3 signaling pathway is associated with inflammation, oxidative stress, apoptosis and fibrosis formation (37-40). Cao et al. found that JAK2/ STAT3 mediated NLRP3 inflammasome suppression in LPS-induced ALI. Inhibiting this pathway could alleviate ALI (9). Kong et al. reported that inhibiting JAK2/STAT3 pathway could alleviate LPS-induced A549 cell injury and apoptosis (41). In the present study, the activation of the JAK2/STAT3 pathway was observed in BLM-induced ALI. Ani could inhibit this pathway by affecting JAK2/STAT3 phosphorylation. To the best of our knowledge, the role of Ani on the JAK2/STAT3 pathway has not been previously determined. Our results indicated that Ani was a potential inhibitor of the JAK2/STAT3 pathway. AG490 is a wellknown JAK2 inhibitor $(30,37)$. Therefore, to further detect the role of Ani on the JAK2/STAT3 pathway, AG490 was used in the present study. Compared with AG490 alone, the stronger inhibitory effect of AG490 combined with Ani on the JAK2/STAT3 pathway was observed. This result further indicated that Ani contributed to the inactivation of JAK2/ STAT3. Furthermore, compared with BLM-induced rats, rats treated with AG490 had lower apoptotic rate, lower levels of fibrosis markers, increased levels of antioxidant enzymes, and decreased levels of pro-inflammatory factors. The effects of AG490 were further reinforced by Ani, indicating Ani could inhibit inflammation, oxidative stress, apoptosis and fibrosis formation in BLM-induced ALI models via inhibiting the JAK2/STAT3 pathway, thus exhibiting a protective effect against ALI.

The present study had several limitations. First, the dose of BLM used in our study was determined by previous reports $(22,23)$. We did not include different groups based on alternate doses to determine the optimal dose of BLM for establishing ALI models in immature rats. Second, in the present study, we found that Ani exhibited a protective effect on BLM-induced ALI via inhibiting the JAK2/STAT3 pathway. However, the effect of Ani on other signaling pathways involved in BLM-induced ALI, such as TLR4/ MyD88/NF-кB (28) and Nrf2/HO-1 (27) pathways, were not detected in the present study. Additionally, the toxic effect of Ani for ALI treatment in immature rats was not observed. Therefore, further studies are warranted.

\section{Conclusions}

Our findings indicated that Ani exhibited a protective effect against BLM-induced ALI in immature rats through inhibiting the JAK2/STAT3 signaling pathway.

\section{Acknowledgments}

Funding: None.

\section{Footnote}

Reporting Checklist: The authors have completed the ARRIVE reporting checklist. Available at http://dx.doi. org/10.21037/atm-21-1750

Data Sharing Statement: Available at http://dx.doi. org/10.21037/atm-21-1750

Conflicts of Interest: All authors have completed the ICMJE uniform disclosure form (available at http://dx.doi. org/10.21037/atm-21-1750). The authors have no conflicts of interest to declare.

Ethical Statement: The authors are accountable for all aspects of the work in ensuring that questions related to the accuracy or integrity of any part of the work are appropriately investigated and resolved. Experiments were performed under a project license (No.: SYXK2018-0015) granted by Institutional Animal Care and Use Committee of Jilin University, in compliance with the Guide for the Care and Use of Laboratory Animals (NIH Publication 86-23, National Academic Press, Washington, DC, USA, 1996).

Open Access Statement: This is an Open Access article distributed in accordance with the Creative Commons Attribution-NonCommercial-NoDerivs 4.0 International License (CC BY-NC-ND 4.0), which permits the noncommercial replication and distribution of the article with the strict proviso that no changes or edits are made and the original work is properly cited (including links to both the formal publication through the relevant DOI and the license). See: https://creativecommons.org/licenses/by-nc-nd/4.0/.

\section{References}

1. Zhou T, Chen YL. The Functional Mechanisms of miR- 
30b-5p in Acute Lung Injury in Children. Med Sci Monit 2019;25:40-51.

2. Labib JR, Ibrahem SK, Sleem HM, et al. Diagnostic indicator of acute lung injury for pediatric critically ill patients at a tertiary pediatric hospital. Medicine 2018;97:e9929.

3. Yu X, Li C. Protective effects of propofol on experimental neonatal acute lung injury. Mol Med Rep 2019;19:4507-13.

4. Dong $Z$, Yuan Y. Accelerated inflammation and oxidative stress induced by LPS in acute lung injury: Inhibition by ST1926. Int J Mol Med 2018;41:3405-21.

5. Zhou Y, Hao C, Li C, et al. Omentin-1 protects against bleomycin-induced acute lung injury. Mol Immunol 2018;103:96-105.

6. Matute-Bello G, Frevert CW, Martin TR. Animal models of acute lung injury. Am J Physiol Lung Cell Mol Physiol 2008;295:L379-399.

7. Lei J, Wei Y, Song P, et al. Cordycepin inhibits LPSinduced acute lung injury by inhibiting inflammation and oxidative stress. Eur J Pharmacol 2018;818:110-4.

8. Meng L, Zhao X, Zhang H. HIPK1 Interference Attenuates Inflammation and Oxidative Stress of Acute Lung Injury via Autophagy. Med Sci Monit 2019;25:827-35.

9. Cao F, Tian X, Li Z, et al. Suppression of NLRP3 Inflammasome by Erythropoietin via the EPOR/JAK2/ STAT3 Pathway Contributes to Attenuation of Acute Lung Injury in Mice. Front Pharmacol 2020;11:306.

10. Sahu B, Narota A, Naura AS. Pharmacological inhibition of poly (ADP-ribose) polymerase by olaparib, prevents acute lung injury associated cognitive deficits potentially through suppression of inflammatory response. Eur J Pharmacol 2020;877:173091.

11. Kheiry M, Dianat M, Badavi M, et al. p-Coumaric Acid Attenuates Lipopolysaccharide-Induced Lung Inflammation in Rats by Scavenging ROS Production: an In Vivo and In Vitro Study. Inflammation 2019;42:1939-50.

12. Yang H, Hua C, Yang X, et al. Pterostilbene prevents LPSinduced early pulmonary fibrosis by suppressing oxidative stress, inflammation and apoptosis in vivo. Food Funct 2020;11:4471-84.

13. Valavanidis A, Vlachogianni T, Fiotakis K, et al. Pulmonary oxidative stress, inflammation and cancer: respirable particulate matter, fibrous dusts and ozone as major causes of lung carcinogenesis through reactive oxygen species mechanisms. Int J Environ Res Public
Health 2013;10:3886-907.

14. Qin MZ, Qin MB, Liang ZH, et al. Effect of SOCS3 on lung injury in rats with severe acute pancreatitis through regulating JAK2/STAT3 signaling pathway. Eur Rev Med Pharmacol Sci 2019;23:10123-31.

15. Yu Y, Zhu T, Li Y, et al. Repeated intravenous administration of silica nanoparticles induces pulmonary inflammation and collagen accumulation via JAK2/STAT3 and TGF- $\beta /$ Smad 3 pathways in vivo. Int J Nanomedicine 2019;14:7237-47.

16. Zhang L, Lu P, Guo X, et al. Inhibition of JAK2/ STAT3 signaling pathway protects mice from the DDPinduced acute kidney injury in lung cancer. Inflamm Res 2019;68:751-60.

17. Yin XL, Shen H, Zhang W, et al. Inhibition of endoplasm reticulum stress by anisodamine protects against myocardial injury after cardiac arrest and resuscitation in rats. Am J Chin Med 2011;39:853-66.

18. Yao BJ, He XQ, Lin YH, et al. Cardioprotective effects of anisodamine against myocardial ischemia/ reperfusion injury through the inhibition of oxidative stress, inflammation and apoptosis. Mol Med Rep 2018;17:1253-60.

19. Li YF, Xu BY, An R, et al. Protective effect of anisodamine in rats with glycerol-induced acute kidney injury. BMC Nephrol 2019;20:223.

20. Guoshou Z, Chengye Z, Zhihui L, et al. Effects of high dose of anisodamine on the respiratory function of patients with traumatic acute lung injury. Cell Biochem Biophys 2013;66:365-9.

21. You QH, Zhang D, Niu CC, et al. Expression of IL$17 \mathrm{~A}$ and IL-17F in lipopolysaccharide-induced acute lung injury and the counteraction of anisodamine or methylprednisolone. Cytokine 2014;66:78-86.

22. Tsukioka T, Takemura S, Minamiyama Y, et al. Attenuation of Bleomycin-Induced Pulmonary Fibrosis in Rats with S-Allyl Cysteine. Molecules 2017;22:543.

23. Robbe A, Tassin A, Carpentier J, et al. Intratracheal Bleomycin Aerosolization: The Best Route of Administration for a Scalable and Homogeneous Pulmonary Fibrosis Rat Model? Biomed Res Int 2015;2015:198418.

24. Lv H, Liu Q, Sun Y, et al. Mesenchymal stromal cells ameliorate acute lung injury induced by LPS mainly through stanniocalcin-2 mediating macrophage polarization. Ann Transl Med 2020;8:334.

25. Thompson BT, Chambers RC, Liu KD. Acute Respiratory Distress Syndrome. N Engl J Med 2017;377:562-72. 
26. Getsy PM, Mayer CA, MacFarlane PM, et al. Acute lung injury in neonatal rats causes postsynaptic depression in nucleus tractus solitarii second-order neurons. Respir Physiol Neurobiol 2019;269:103250.

27. Dang JW, Lei XP, Li QP, et al. Asiaticoside attenuates hyperoxia-induced lung injury in vitro and in vivo. Iran J Basic Med Sci 2019;22:797-805.

28. Zhao J, Bi W, Zhang J, et al. USP8 protects against lipopolysaccharide-induced cognitive and motor deficits by modulating microglia phenotypes through TLR4/MyD88/ $\mathrm{NF}-\kappa \mathrm{B}$ signaling pathway in mice. Brain Behav Immun 2020;88:582-96

29. Song H, Chen Q, Xie S, et al. GDF-15 prevents lipopolysaccharide-mediated acute lung injury via upregulating SIRT1. Biochem Biophys Res Commun 2020;526:439-46.

30. Pinto MP, Balmaceda C, Bravo ML, et al. Patient inflammatory status and CD4+/CD8+ intraepithelial tumor lymphocyte infiltration are predictors of outcomes in high-grade serous ovarian cancer. Gynecol Oncol 2018;151:10-7.

31. Li Q, Li B, Liu C, et al. Protective role of Nrf2 against mechanical-stretch-induced apoptosis in mouse fibroblasts: a potential therapeutic target of mechanical-traumainduced stress urinary incontinence. Int Urogynecol J 2018;29:1469-77.

32. Pan S, Chen Y, Zhang X, et al. The JAK2/STAT3 pathway is involved in dexmedetomidine-induced myocardial protection in rats undergoing cardiopulmonary bypass. Ann Transl Med 2020;8:483.

33. Luo L, Zhou A. Antifibrotic activity of anisodamine in vivo is associated with changed intrahepatic levels of matrix metalloproteinase-2 and its inhibitor tissue inhibitors of metalloproteinases- 2 and transforming growth factor beta 1 in rats with carbon tetrachloride-induced liver injury. J

Cite this article as: Zhao X, Zhao B, Zhao Y, Zhang Y, Qian M. Protective effect of anisodamine on bleomycin-induced acute lung injury in immature rats via modulating oxidative stress, inflammation, and cell apoptosis by inhibiting the JAK2/STAT3 pathway. Ann Transl Med 2021;9(10):859. doi: 10.21037/atm-211750
Gastroenterol Hepatol 2009;24:1070-6.

34. Mou F, Mou C. Necrostatin-1 Alleviates BleomycinInduced Pulmonary Fibrosis and Extracellular Matrix Expression in Interstitial Pulmonary Fibrosis. Med Sci Monit 2020;26:e919739.

35. Geng Z, Fan WY, Zhou B, et al. FNDC5 attenuates obesity-induced cardiac hypertrophy by inactivating JAK2/ STAT3-associated inflammation and oxidative stress. J Transl Med 2019;17:107.

36. Owais K, Huang T, Mahmood F, et al. Cardiopulmonary Bypass Decreases Activation of the Signal Transducer and Activator of Transcription 3 (STAT3) Pathway in Diabetic Human Myocardium. Ann Thorac Surg 2015;100:1636-45.

37. Zhou Y, Sun Y, Hou W, et al. The JAK2/STAT3 pathway inhibitor, AG490, suppresses the abnormal behavior of keloid fibroblasts in vitro. Int J Mol Med 2020;46:191-200.

38. Zhang Y, Liu J, Yang B, et al. Ginkgo biloba Extract Inhibits Astrocytic Lipocalin-2 Expression and Alleviates Neuroinflammatory Injury via the JAK2/ STAT3 Pathway After Ischemic Brain Stroke. Front Pharmacol 2018;9:518.

39. Milara J, Hernandez G, Ballester B, et al. The JAK2 pathway is activated in idiopathic pulmonary fibrosis. Respir Res 2018;19:24.

40. Cao G, Zhu R, Jiang T, et al. Danshensu, a novel indoleamine 2,3-dioxygenase 1 inhibitor, exerts anti-hepatic fibrosis effects via inhibition of JAK2-STAT3 signaling. Phytomedicine 2019;63:153055.

41. Kong F, Sun Y, Song W, et al. MiR-216a alleviates LPSinduced acute lung injury via regulating JAK2/STAT3 and NF-кB signaling. Hum Cell 2020;33:67-78.

(English Language Editor: R. Scott) 